\title{
Endpoints of rotation intervals for maps of the circle
}

\author{
GRZEGORZ ŚWIASTEK \\ Institute of Mathematics, Department of Mathematics, PKiN, 00-901 Warsaw, Poland
}

(Received 20 January 1986, revised 14 October 1987)

\begin{abstract}
For certain $C^{2}$ one-parameter families of endomorphisms of the circle both endpoints of rotation intervals are rational except for a set of parameter values of zero Lebesgue measure.
\end{abstract}

\section{Introduction}

Families like

$$
(x) H_{\lambda}: S^{1} \rightarrow S^{1}, \quad H_{\lambda}(x)=x+c \sin (2 \pi x)+\lambda(\bmod 1)
$$

have been widely studied for their relevance to the existence of invariant circles of various irrational rotation numbers in the KAM theory, [2]. For $|c|<1 / 2 \pi$ this is a family of diffeomorphisms of the circle and these have irrational rotation numbers for a set of parameter values $\lambda$ of positive Lebesgue measure. If $c=1 / 2 \pi$ the maps are homeomorphisms but not diffeomorphisms and it is believed on numerical evidence, see [5] that such parameter values have measure zero. When $c>1 / 2 \pi$ the maps are not homeomorphisms and may have a rotation interval rather than a rotation number.

In this paper we deal with the endomorphisms of the circle which are not homeomorphisms. The notion of rotation set for such maps is the generalization of the concept of rotation number for homeomosphisms. It was introduced in [7] where the proof that the rotation set is always an interval can also be found. The question then arose concerning how the endpoints of rotation intervals vary when oneparameter families of endomorphisms of the circle are considered? By analogy with the already quoted result for diffeomorphisms (see [4]) it was conjectured that if endpoints really vary with the parameter then at least one of them is irrational for a set of parameter values of positive Lebesgue measure. On the other hand, the results of [1] suggested that this measure was zero for piecewise linear maps. Our result goes along that line. We shall prove that the endpoints of the rotation intervals are rational for almost every parameter value for certain $C^{2}$ families of maps. Indeed these maps form a set with non-empty interior in the $C^{2}$ topology.

However, this set is not dense, so it may happen that the conjecture is true for an open non-empty set of families.

I express my thanks to M. Misiurewicz who suggested the subject of this paper and carefully read the manuscript. 


\section{Formulation of the theorem}

We shall provide 2 sets of assumptions (called Version 1 and Version 2, abbreviated to V1 and V2 respectively). The claim is the same in both cases, namely that

The upper endpoint of the rotation interval is rational for almost all parameter values in the sense of Lebesgue measure.

Version 1 is rather complicated, but general. Version 2 is much simpler, but can only be applied to very particular families. It seems that our hypotheses indicate the limitations of our method rather than they really describe the set of families for which the above claim is true. In the actual proof we shall use the technical set of assumptions, called the Local Version (LV), which will be formulated later. Version 2 can be used to produce a lot of specific examples, in particular it also comprises the family $(*)$ discussed in the introduction for $c>1 / 2 \pi$. A reader who does not want to go deeply into details may trace the proof in the special case of the family (*). It is easy to verify that it satisfies V2 with $\alpha=1 / 2 \pi \operatorname{arc} \cos (-1 / 2 \pi c)$.

We assume that we have fixed an orientation of the circle. Everywhere we speak of lifts we mean lifts to the coverings of $S^{1}$ by $\mathbb{R}^{1}$, which agree with this orientation.

Precisely, the rotation set depends on lift, but the property whether its upper endpoint is rational or not does not depend on which lift from the range indicated above we have chosen. If we change the orientation fixed on $S^{\mathbf{l}}$, then the endpoints of the rotation interval are interchanged. This is the way we extend our result onto the lower endpoint of the rotation interval. However, for various orientations we usually get different families of lifts and our hypotheses must be checked for either of them separately. The family $(* *): x \mapsto \lambda \sin (2 \pi x)+x(\bmod 1), \lambda>1 / 2 \pi$, satisfies V1; moreover so do families from some part of its open neighbourhood. Also observe that the family of lifts with respect to the reversed orientation is described by the same formula (since it is an odd function). Thus we obtain an example of a non-empty, open set of families for which V1 holds with respect to both orientations, hence both endpoints of rotation intervals are rational almost everywhere.

We pass to the formulation of our hypotheses.

We study families of degree one maps of the circle $H_{\lambda}: S^{1} \rightarrow S^{1}$ depending on the real parameter $\lambda$. All $H_{\lambda}$ are not homeomorphisms. We write $H(\lambda, x)$ for $H_{\lambda}(x)$ so that, for some open interval $U \subset \mathbb{R}, H$ maps $U \times S^{1} \rightarrow S^{1}$. (H is assumed at least continuous.) Then $h: U \times \mathbb{R} \rightarrow \mathbb{R}$ will denote a continuous family of lifts of maps $H$.

Our hypotheses are designed to ensure that our maps $h$ are differentiable, nondegenerate, increasing with parameter and one-to-one and diffeomorphisms on some large interval. The detailed assumptions may seem a little bit artificial - it is useful to also have in mind that they should guarantee that upper maps have exactly 1 modulo 1 interval, on which they are constant and that they increase with the parameter for each $x$ (see $\$ 3$ for a definition and some remarks on upper maps).

Version 1

(1) The function $H$ is $C^{2}$.

The remaining hypotheses are formulated in terms of lifts $h$. This dependence on the lifts is only formal, however, since they hold for each lift once they hold for one of them. 
We claim that for each $\lambda \in U$ there exists $\alpha_{\lambda} \in \mathbb{R}$ such that the following conditions are satisfied:

(2) The point $\alpha_{\lambda}$ is a strict local maximum of $h_{\lambda}$ and also a strict global maximum of $h_{\lambda}$ restricted to $\left(-\infty, \alpha_{\lambda}\right]$.

We also demand that some $\beta_{\lambda}$ should exist such that for each $\lambda \in U$ the following hypotheses are satisfied:

(3) (a) $\beta_{\lambda}$ is a (not strict) maximum of $h_{\lambda}$ restricted to $\left(-\infty, \beta_{\lambda}\right]$,

(b) $h_{\lambda}\left(\beta_{\lambda}\right)=h_{\lambda}\left(\alpha_{\lambda}\right)-1$,

(c) $\partial h_{\lambda} / \partial x$ is positive on $\left[\beta_{\lambda}, \alpha_{\lambda}\right)$.

(4) The derivative $\partial h / \partial \lambda$ is positive at each point $(\lambda, x) \in U \times \mathbb{R}$ such that $\beta_{\lambda} \leqq x \leqq$ $\alpha_{\lambda}$.

(5) $\left(d^{2} h_{\lambda} / \mathrm{d} x^{2}\right)\left(\alpha_{\lambda}\right)<0$.

After Version 1 has been formulated, we are going to present some remarks on it. In particular, we want to explain the dependence of $\alpha_{\lambda}$ and $\beta_{\lambda}$ on the parameter. See also figure 1, which shows an example of a lift $h_{\lambda}$.

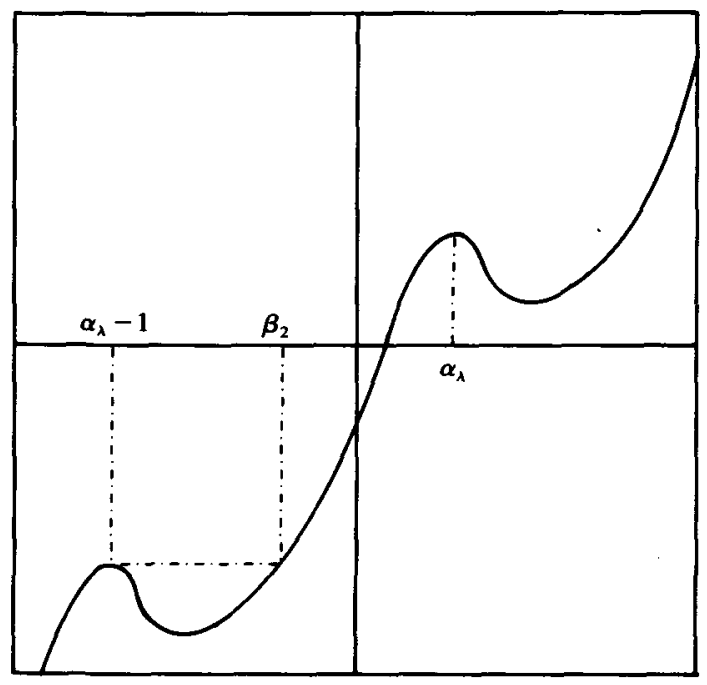

FIGURE 1

It is clear that, since $h_{\lambda}$ are lifts of maps of the circle, if there is one pair $\alpha_{\lambda}, \beta_{\lambda}$ satisfying V1 for some $\lambda$, then there are infinitely many such pairs. We shall prove, however, that modulo 1 there is only one such pair for each parameter value. This uniqueness will persist even if we weaken the condition (3)(b) demanding only that $h_{\lambda}\left(\alpha_{\lambda}\right)-h_{\lambda}\left(\beta_{\lambda}\right)>\frac{1}{2}$. Suppose that $\alpha_{\lambda}$ satisfies the weakened assumptions and let us try to fulfil them with another $a \in\left(\alpha_{\lambda}-1, \alpha_{\lambda}\right)$ and respectively, $b$. The derivative of $h_{\lambda}$ must vanish at $a$ as well as at $\alpha_{\lambda}-1$ since they are local maxima. Thus it must be $\alpha_{\lambda}-1<b<a<\alpha_{\lambda}$, otherwise (3)(c) would be violated. Since $h_{\lambda}\left(\alpha_{\lambda}-1\right)=$ $h_{\lambda}\left(\alpha_{\lambda}\right)-1$, the weakened $(3)(b)$ condition implies that either $h_{\lambda}\left(\beta_{\lambda}\right)$ is less than $h_{\lambda}(a)$ or $h_{\lambda}(b)$ is less than $h_{\lambda}\left(\alpha_{\lambda}-1\right)$ - both possibilities contradict (3)(a). Thus we 
have established the uniqueness modulo 1 of $\alpha_{\lambda}$ and it is trivial that $\alpha_{\lambda}$ uniquely determines $\beta_{\lambda}$. It makes sense to regard $\alpha_{\lambda}$ and $\beta_{\lambda}$ as functions of $\lambda$. We shall prove that these functions are $C^{1}$.

Once more we only have a problem with $\alpha_{\lambda}$, since the dependence of $\beta_{\lambda}$ on $\alpha_{\lambda}$ is evidently $C^{2}$. Let us fix some $\lambda_{0}$ in $U$ and consider equation $\partial h / \partial x=0$ defined in $U \times \mathbb{R}$. By (5), we may use the implicit function theorem to find that, there exists a solution $x(\lambda)$ defined on some neighbourhood of $\lambda_{0}, C^{1}$ and unique on it. Thus in particular $x\left(\lambda_{0}\right)=\alpha_{\lambda_{0}}$. It is a little work to verify that, when we try to satisfy V1 with the weakened (3)(b) with $\alpha_{\lambda}=x(\lambda)$, it is actually fulfilled, perhaps for $\lambda$ from a still smaller neighbourhood of $\lambda_{0}$. By the uniqueness property we have proved above it implies that $x(\lambda)$ is equal to $\alpha_{\lambda}$ for $\lambda$ from that neighbourhood.

Now we shall write down the hypotheses of Version 2. It is similar to Version 1 and the general assumptions are the same. We assume that the dependence on the parameter has a very particular form, which allows us to significantly simplify the assumptions and also weaken the requirements concerning differentiability with respect to the argument on the circle. We formulate our assumptions in terms of lifts.

\section{Version 2}

There exists a function $M: \mathbb{R} \rightarrow \mathbb{R}$ such that $h_{\lambda}(x)=M(x)+\lambda$ is a family of lifts of $H$ (it implies in particular that $M(x+1)=M(x)+1$, so that $M$ is an 'old map'). We also assume the following:

(1) There is $\alpha \in \mathbb{R}$ such that $\alpha$ is a local maximum of $M$ and a strict global maximum of $M_{\mid(\rightarrow \infty, \alpha)}$.

(2) There is $\beta \in \mathbb{R}$ with $M(\beta)=M(\alpha)-1$ such that $\beta$ is a (not strict) global maximum of $M_{\mid(-\infty, \beta]}$.

We also assume a few differentiability properties of $M$ on the interval $(\beta, \alpha)$, namely:

(3) The function $M$ is $C^{2}$ on that interval with bounded derivatives (in fact $C^{1}$ with a bounded variation of the derivative would suffice).

(4) $\mathrm{d} M / \mathrm{d} x$ is positive on $(\beta, \alpha)$ and may tend to 0 only at $\alpha$.

(5) There is $\varepsilon \in(\beta, \alpha)$ such that $\mathrm{d} M / \mathrm{d} x$ is non-increasing on $(\varepsilon, \alpha)$.

We would like to emphasize that we have assumed nothing about the differentiability of $M$ outside $(\beta, \alpha)$. This is due to the fact that we replace functions $h$ by their upper maps in the actual proof. We shall discuss this in detail in the next section.

\section{Upper maps and the local version}

The upper map $u: \mathbb{R} \rightarrow \mathbb{R}$ for a given map $v: \mathbb{R} \rightarrow \mathbb{R}$ being a lift of some continuous endomorphism of the circle is defined by: $u(x)=\sup \{v(y): y \leqq x\}$. You can see this transformation in figure 2 . Note that $u$ is non-decreasing and is a lift of some weakly order preserving map of the circle of degree one. Such maps have a well-defined rotation number. There is a theorem which says that the rotation number of $u$ is equal to the upper endpoint of the rotation interval of $v$. (For the proof see for example [6].) 


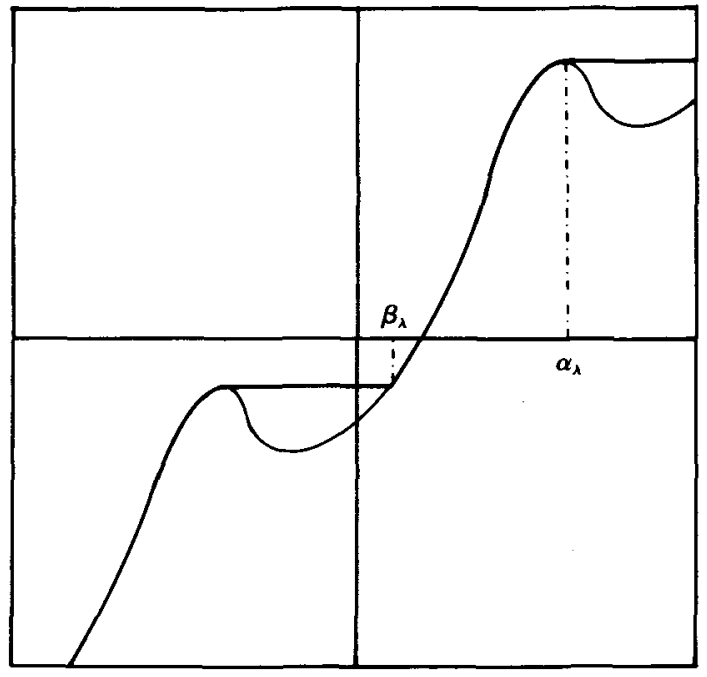

Figure 2

Now we replace the lifts $h$ of V1 and V2 by their upper maps denoted by $f$. This explains why there is no differentiability assumed for $M$ outside $(\beta, \alpha)$ in V2. The upper map is constant outside this interval. We shall furnish the set of assumptions called the Local Version (LV) which are sufficient to guarantee that a family of weakly order preserving maps of the circle has a rational rotation number for almost every parameter value. It is the proof of $\mathrm{LV}$ that is really difficult. We shall also show - and it is easy - that if either V1 or V2 holds for a given family $H$ then LV holds for upper maps $f$ provided you choose lifts $h$ and the parameter space $T$ properly. $T$ is an open interval, usually smaller than $U$, but we shall be able to overcome this difficulty using some countable covering argument.

\section{Local version}

We deal with the families $F_{\lambda}$ of weakly order preserving, degree one maps of the circle depending on a real parameter $\lambda$ which belongs to some open inteval $T$. In the natural way we obtain the mapping $F: T \times S^{1} \rightarrow S^{1}$ given by $F(\lambda, x)=F_{\lambda}(x)$. We assume $F$ to be at least continuous and by $f$ we denote a continuous family of lifts of maps $F$.

Our assumptions are formulated in terms of $f$. They state that $f$ should be differentiable and increasing with the parameter and non-decreasing with respect to the other argument. The graph of each $f_{\lambda}$ should look as figure 3 shows - with an exactly one modulo 1 flat 'shelf' and with a positive slope between the shelves tending to 0 only on one side.

Such maps have a rotation number which we denote by $\rho(\lambda)$ for each $\lambda$. The claim of $L V$ is that the set $\rho^{-1}(\mathbb{R} \backslash Q)$ is of zero Lebesgue measure. 


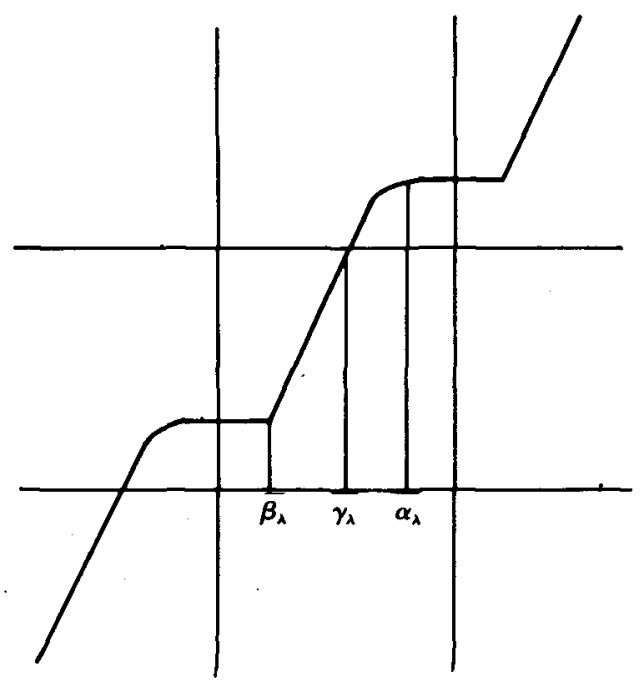

Figure 3

The detailed assumptions are as follows:

(1) For each $\lambda \in T$ the rotation number $\rho(\lambda)$ is between 0 and 1. We define:

$$
\begin{aligned}
& \alpha_{\lambda}=\min \left\{x \in[0,1]: \forall y \in[0,1] f_{\lambda}(y) \leq f_{\lambda}(x)\right\} \quad \text { and } \\
& \beta_{\lambda}=\max \left\{x \in[0,1]: f_{\lambda}(x)=f_{\lambda}\left(\alpha_{\lambda}\right)-1\right\} .
\end{aligned}
$$

Then we also have:

(2) There exists a constant $\alpha$ with $\alpha_{\lambda} \leq \alpha<1$ for each $\lambda \in T$.

Let us define the set $\Gamma$ :

$$
\Gamma=\left\{(\lambda, x) \in T \times(0,1): x \in\left(\beta_{\lambda}, \alpha_{\lambda}\right) .\right.
$$

(3) The function $f$ is $C^{2}$ when restricted to $\Gamma$ with all derivatives bounded by some constant $L$. We also assume that $L|T|<\frac{1}{2}$.

(4) There is such a positive constant $\sigma$ that $\partial f / \partial \lambda \geq \sigma$ on $\Gamma$.

(5) Let $g: T \rightarrow \mathbb{R}$ be defined by $g(\lambda)=f_{\lambda}(0)$. Then $g$ is $C^{1}$ with $\mathrm{d} g / \mathrm{d} \lambda \geq \sigma$.

(6) There is a function $\gamma: T \rightarrow(0,1)$ whose value at $\lambda$ is denoted by $\gamma_{\lambda}$ such that:

(a) For each $\lambda \in T$ the derivative $\partial f_{\lambda} / \partial x$ is non-increasing on $\left(\gamma_{\lambda}, \alpha_{\lambda}\right)$.

(b) There exists a positive constant $\zeta$ such that $\partial f / \partial x \geq \zeta$ on the set $\left\{(\lambda, x): \beta_{\lambda}<x \leq \gamma_{\lambda}\right\}$.

Now we shall give some ideas of the proof that for each $\vec{\lambda} \in U$ such that the upper endpoint of rotation interval of $\lambda_{\bar{\lambda}}$ is not an integer, there is an open interval $T$ with $\bar{\lambda} \in T \subset U$ on which LV is satisfied for $f_{\lambda}$ being upper maps for the suitably chosen lifts of $H$.

We start with any family of lifts $h$ and we are going to adjust it in two steps in order to obtain the 'suitably chosen' family (which is not unique). First, we can ensure that $\rho\left(f_{\bar{\lambda}}\right)$ is between 0 and 1 - simply by replacing lifts $h_{\bar{\lambda}}$ by $h_{\bar{\lambda}}-m$, where $m$ is the integer part of the upper endpoint of the rotation interval for $h_{\bar{\lambda}}$. If we do it also for $\lambda$ sufficiently close to $\bar{\lambda}$, then the upper maps obtained for such lifts satisfy (1)LV with $T$ some neighbourhood of $\bar{\lambda}$ ). Here we have used the fact that 
$\rho$ is continuous with respect to $\lambda$, which was proved in [2]. The new family of lifts will also be denoted by $h$.

The goal of the next adjustment is to obtain lifts for which the $\alpha_{\lambda}$ and $\beta_{\lambda}$ of Version 1 or Version 2 are in the interval $[0,1]$ (we put $\alpha_{\lambda}$ and $\beta_{\lambda}$ equal identically to $\alpha$ and $\beta$ in the case of Version 2). To avoid ambiguity of notations we shall mark $\alpha_{\lambda}$ and $\beta_{\lambda}$ in the sense of LV with bars. It is easy to see that we can choose such a positive number $c$ and $T$ an interval containing $\bar{\lambda}$ that $\alpha_{\lambda}$ is a strict global maximum of $h_{\lambda}$ restricted to $\left(-\infty, \alpha_{\lambda}+c\right]$ and $\left|\alpha_{\lambda}-\alpha_{\bar{\lambda}}\right|<c / 2$ for all $\lambda \in T$ (to see this it is necessary to recall that $\alpha_{\lambda}$ is continuous in $\lambda$ ). Next, we displace graphs of $h_{\lambda}$ for $\lambda \in T$ by the vector $v=\left(1-\alpha_{\lambda}-c, 1-\alpha_{\lambda}-c\right)$. Figure 4 shows what happens. The shifted graphs define the new family of lifts also denoted by $h_{\lambda}$ and it is already an appropriate one. Such a change is equivalent to moving the point of $\mathbb{R}^{1}$, which is mapped to 1 by the projection defining lifts $h$ (we make the identification $S^{1}=$ $\left.\left\{\mathrm{e}^{\mathrm{i} t}: t \in \mathbb{R}\right\}\right)$. It is also clear that all the properties assumed in Version 1 or Version 2 remain undisturbed. Now $\bar{\alpha}_{\lambda}=\alpha_{\lambda}$ and $\bar{\beta}_{\lambda}=\beta_{\lambda}$ so that we may remove the bars from further notations.

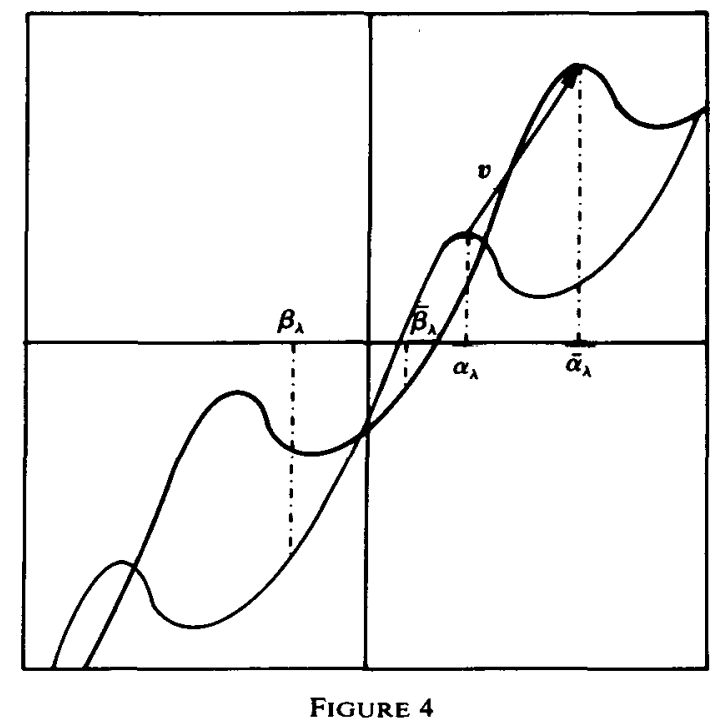

Observe now that $f_{\lambda}$ coincides with $h_{\lambda}$ on $\Gamma$. It allows us to verify the rest of LV in a simple way. It is even quite obvious when we start from Version 2. Thus we shall concentrate on V1 only. The assumptions (1) and (2) follow from the above construction. In (3) LV one needs only to prove that the derivatives are bounded. To manage it we can take $T^{\prime}$ cointained with its closure in $T$ and then use the compactness argument in order to obtain the boundedness of derivatives on $T^{\prime}$. We must perhaps take $T^{\prime}$ still smaller in order to obtain $L\left|T^{\prime}\right|<\frac{1}{2}$. A similar argument will also work in (4) LV and (6)(b)LV. In (5) we have $g(\lambda)=f_{\lambda}\left(\alpha_{\lambda}\right)-1$, thus

$$
\frac{d g}{d \lambda}=\frac{\partial f}{\partial x}\left(\lambda, \alpha_{\lambda}\right) \frac{d a_{\lambda}}{d \lambda}+\frac{\partial f}{\partial \lambda}\left(\lambda, \alpha_{\lambda}\right)=\frac{\partial f}{\partial \lambda}\left(\lambda, \alpha_{\lambda}\right) \geq \sigma \text {. }
$$


To justify this computation recall that $\alpha_{\lambda}$ depends differentiably on $\lambda$. To satisfy (6) $\mathrm{LV}$ is a rather more delicate thing. By (5) V1 the second derivative of $h_{\lambda}$ is negative at $\alpha_{\lambda}$. It is also negative on some open neighbourhood of the curve $\left\{\left(\lambda, \alpha_{\lambda}\right): \lambda \in U\right\}$ in $U \times \mathbb{R}$. Also an open and convex neighbourhood $w$ with this property can be obtained - perhaps we must confine $T$ and use a compactness argument. A similar argument shows also that $\partial f / \partial x$ is separated from 0 on the complement of this neighbourhood in $\Gamma$. Thus we can satisfy (6)LV putting $\gamma_{\lambda}=\sup \{x \in \mathbb{R}:(\lambda, x) \in$ $W \cap \Gamma\}$.

We have at least sketched the proof that it is possible to cover the set $U$ except for tie set of parameter values corresponding to integer values of upper endpoint of rotation interval with intervals $T$, on which LV is satisfied. It is an elementary topological fact that we can choose only a countable number of such intervals, which also cover this set (it is an open set, since $\rho$ is continuous). Thus the claim of both Version 1 and Version 2 follows immediately from the claim of LV.

The rest of this paper will be devoted to the proof of the theorem under LV assumptions.

\section{An outline of the proof of the local theorem}

In $\S 4$ we begin with a study of the very simple family of maps - namely the rotations by $2 \pi x$ where $x$ is a parameter. Lifts of these maps are simply translations by $x$ on the real line. We investigate the distribution of the orbit of 0 by the translation by $x$. We show that it is related to the continued fraction representation of $x$.

In $\S 6$ we expand the results of the previous section on the family $f$. It is based on the existence of topological semi-conjugacy between maps with the same irrational rotation number. Since the results of $\S 5$ concern the ordering of the orbits of 0 , they are valid also for maps $f$, at least those with an irrational rotation number. For any rational number $u$ we define the set $I(u)$ in the parameter space. The definition is not very simple, but in essence $I(u)$ consists of the parameters for which the continued fraction representation of the rotation number of $f$ has some odd convergent equal to $u$. We shall prove that the situation looks as shown by figure 5 . We assume that $u=p / q$ with $(p, q)=1$ and the arrow means the function which associates with every parameter value $\lambda$ the $q$ th image of 0 by $f_{\lambda}$. We can see that some upper part of $I(u)$, called $K(u)$, goes to $(\alpha+p-1, p)$, that is under the shelf of the graph of $f$. It is clear that for parameters from $K(u)$ the rotation number must be $u$. In $\S 6$ we prove that the length of the image of $K(u)$ by the function marked with the arrow cannot be too small as related to the image of the whole $I(u)$ by the same function. If we knew that the arrow cannot distort $I(u)$ too much, we would obtain that $K(u)$ is not too small as related to $I(u)$. In $\S 6$ we prove that the intervals $\left\{f_{\lambda}^{i}(0): \lambda \in I(u)\right\}$ are disjoint modulo 1 for $I$ not exceeding $q$. Moreover for $i$ less than $q$ they are always under the non-flat part of the graph of $f_{\lambda}$. (Suppose for a moment that we have Version 2 with $\alpha_{\lambda}$ and $\beta_{\lambda}$ independent of the parameter.) Then if the variation of $\log d f_{\lambda} / d x$ were bounded on $\left(\beta_{\lambda}, \alpha_{\lambda}\right)$ for every $\lambda$ the distortion could be estimated by a method similar to that of Denjoy's proof of the non-existence of homtervals for diffeomorphisms of the circle with irrational rotation 


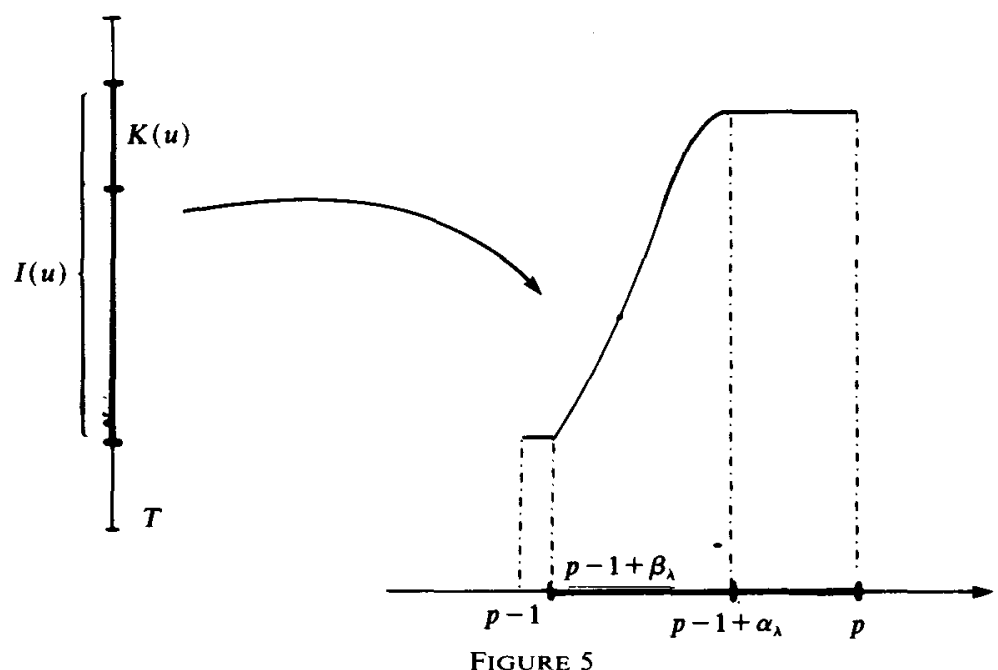

number. This is not our case, since $\mathrm{d} f_{\lambda} / \mathrm{d} x$ may tend to zero at $\alpha_{\lambda}$. However it cannot tend to zero at $\beta_{\lambda}$ and using this fact we prove in $\S 7$ that the ratio $|K(u)| /|I(u)|$ can be reduced very widely by the arrow function but it can grow only by some multiplicative constant. Fortunately, it suffices to prove that, independently of $u$, $|K(u)| /|I(u)|$ is not less than some positive constant $w$. Using this fact we finish the proof of $\mathrm{LV}$ by the simple argument of showing that the set of parameter values with irrational rotation number cannot contain points of density.

\section{Continued fractions and rotation on the circle}

We shall define some numerical sequences for each number $x$ from the interval $(0,1)$. Some of them are closely related to the continued fraction representation. For example, the sequence $b_{n}(x)$ consists of convergents to the continued fraction expansion of $x$ and $q_{n}(x)$ are denominators of irreducible fractions representing $b_{n}(x)$. The nature of $c_{n}(x)$ is different. In fact, it describes the ordering of the orbit of some point on the circle by the rotation by $2 \pi x$. The main goal of this section is to reveal relations between pure arithmetics of continued fractions and some dynamics associated with $c_{n}(x)$. The idea of the use of continued fractions in this problem is not new and was previously used to list intervals in the parameter space corresponding to rational numbers.

Everywhere in this section we assume that $x, u, v$ are numbers from the interval $(0,1)$ and that $u$ and $v$ are rational while $x$ is a real number. Sometimes we shall use expressions like ' $q$-sequence of $x$ ' instead of 'the sequence $q_{n}(x)$ '.

Everywhere in this paper $[\cdot]$ denotes the integral part and $\{\cdot\}$ the fractional part. Definition:

For any $x$ we define the sequences $a_{n}(x)$ and $r_{n}(x)$ inductively as follows:

$$
\begin{gathered}
r_{0}(x)=x, \\
r_{i+1}(x)=\left\{\frac{1}{r_{i}(x)}\right\}, \quad a_{i+1}(x)=\left[\frac{1}{r_{i}(x)}\right] .
\end{gathered}
$$


When $r_{i}(x)$ is 0 for some $i$, the procedure terminates. In that case we obtain the finite sequences, otherwise they are infinite. The $a$-sequence is the sequence of partial quotients of a continued fraction representation of $x$.

Definition. For a given sequence $a_{n}(x)$ we define $b_{k}(x)$ by

$$
b_{k}(x)=\frac{1}{a_{1}(x)+\frac{1}{a_{2}(x)+\cdots+\frac{1}{a_{k-1}(x)+\frac{1}{a_{k}(x)}}}}
$$

where $k$ is assumed to be less than or equal to the length of the $a$-sequence. The numbers $b_{k}(x)$ will be called convergents to the continued fraction representation of $x$ or simply convergents.

Definition. We can write $b_{k}(x)$ in the unique way as $p_{k}(x) / q_{k}(x)$ with $p_{k}(x)$ and $q_{k}(x)$ positive integers and co-prime. It defines the $p$ - and $q$-sequences of $x$.

Now we shall list some properties of the sequences defined above. Proofs can be found for example in [3].

(a) These sequences are finite if and only if $x$ is rational.

(b) The sequence $q_{n}(x)$ strictly increases with $n$ for all $x$.

(c) The numbers $q_{n}(x)$ have the following property:

$\operatorname{dist}(n x, \mathbb{Z})<\min \{\operatorname{dist}(i x, \mathbb{Z}): 1 \leq i \leq n\}$ where dist means the ordinary distance between subsets of $\mathbb{R}^{1}$ (dist $(X, Y)=\inf \{|x-y|: x \in X, y \in Y\}$ ).

This property is in fact characteristic for $q_{n}(x)$ and may also be rephrased in terms of the rotation on the circle. Namely, $q_{n}(x)$ are numbers of the iterations of the rotation by which the image of some $y \in S^{1}$ approaches $y$ closer than ever before.

(d) For $k$ even $x q_{k}(x) \geq p_{k}(x)$ and for $k$ odd $x q_{k}(x) \leq p_{k}(x)$ with the equality if and only if $x=p_{k}(x) / q_{k}(x)$.

(e) For any $x, y \in(0,1)$ if $b_{j}(x)=b_{k}(x)$ then $|j-k| \leq 1$ and if $j=k$ then the sequences $b_{i}(x)$ and $b_{i}(y)$ are equal to the $k$ th place.

Definition. We define $J(u)$ to be the set of numbers from the interval $(0,1)$ whose some odd convergent is equal to $u$.

In view of the properties (a) and (e) it means that there exists some integer $k$ such that the $b$-sequences for all numbers in $J(u)$ coincide. The number $u$ belongs to $J(u)$ if and only if $b_{k}(u)=u$ for $k$ odd.

Definition. For a given $x$ we define its $c$-sequence by

$$
c_{i}(x)=[i x] \text {. }
$$

Now we are going to prove some lemmas which concern the properties of sets $J$.

Lемма 1. Let $p / q$ be an irreducible fraction whose value is between 0 and 1. Suppose also that $p / q$ is in $J(u)$ for some $u$ whose q-sequence contains a number not less than q. Then $u$ is equal to $p / q$.

Proof. By the definition of $J$, the $b$-sequence of $p / q$ must contain $u$. However, the last term of that sequence is $q$ and the claim follows immediately from the property (b). 
LEMMA 2. Let $y$ and $z$ be numbers from the interval $(0,1)$ such that their a-sequences are equal to the $k$ th place. If $x$ belongs to the interval $(y, z)$ then its a-sequence to the $k$ th place is also equal to them and $r_{k}(x)$ and $r_{k}(z)$.

Proof. The proof is inductional with respect to $k$.

For $i=1$ we have

$$
a_{1}(y)=\left[\frac{1}{y}\right] \geq\left[\frac{1}{x}\right]=a_{1}(x) \geq\left[\frac{1}{z}\right]=a_{1}(z)
$$

because the integral part is a non-decreasing function. Hence $a_{1}(x)=a_{1}(y)$. Further we obtain:

$$
\frac{1}{y} \geq \frac{1}{x} \geq \frac{1}{z}
$$

which, together with the above conclusion, imply that

$$
\frac{1}{y}-\left[\frac{1}{y}\right]>\frac{1}{x}-\left[\frac{1}{x}\right]>\frac{1}{y}-\left[\frac{1}{y}\right]
$$

and finally

$$
r_{1}(y)>r_{1}(x)>r_{1}(z) \text {. }
$$

The inductive step is analogous - in the above reasoning we have only used the fact that $r_{0}(x)$ is between $r_{0}(y)$ and $r_{0}(z)$.

LEMMA 3. For each $u$ the set $J(u)$ is an interval.

Proof. Suppose that $s$ and $t$ belong to $J(u)$ and $s<t$. Consider some $x \in(s, t)$. By the definition of $J$ the convergents to continued fractions of $s$ and $t$ are the same as to some $k$ th place. The same must be true of their $a$-sequences by the property (e). Now we apply the previous lemma and obtain that the $a$-sequence of $x$ is also equal to the $a$-sequence of $s$ and $t$ to the $k$ th place. Since their $b$-sequences must be equal to the same place, $b_{k}(x)=u$.

LemMA 4. A rational number $u$ is always the upper endpoint of $J(u)$.

Proof. If $x \in J(u)$ then $b_{k}(x)=u$ for some odd $k$. By the property (d) it implies that $x q_{k}(x) \leq p_{k}(x)$ and thus $x \leq p_{k}(x) / q_{k}(x)=u$. To show that $u$ is really an endpoint we choose $r / s<u=p / q$ with $r q-p s=-1$. It is a property of the Farey series (see [3] that the numbers $(p n+r) /(q n+s)$ are in $J(u)$ and it is clear that they tend to $u$ when $n$ grows to infinity.

LEMMA 5. Both endpoints of $J(u)$ are rational.

Proof. Let us regard $b_{k}(\cdot)$ as a function. It is clear that for every $k$ it is continuous in irrational points. The claim follows immediately.

LeMMA 6. Let us consider the irreducible fraction $p / q$, whose value is $u$. The first $q$ terms are the same for all sequences $c_{n}(x)$ with $x$ belonging to the interior of $J(u)$.

Proof. We shall show that for each natural $i$ not greater than $q$ there exists such natural $m(i)$ that $i($ int $J(u)) \subset(m(i)-1, m(i))$. Otherwise there would be $y \in J(u)$ with $i_{0} y \in \mathbb{N}$ for some natural $i_{0}$ not greater than $q$. We would obtain thus $y=y i_{0} / i_{0}$. By Lemma 1 it follows that $y=p / q$. It contradicts Lemma 4. 
LEMMA 7. For each $u=p / q,(p, q)=1$, and $x$ from the interior of $J(u)$ the following holds:

$$
c_{1}(x)=c_{q+1}(x)-p, \quad c_{2}(x)=c_{q+2}(x)-p, \ldots, \quad c_{q-1}(x)=c_{2 q-1}(x)-p .
$$

Proof. We compare the sequences $p+i x$ and $(q+i) x$. It is clear that $[p+i x]=$ $c_{i}(x)+p$. The sequence $(q+i) x$ differs very little from the sequence $p+i x$, because $|p+i x-(q+i) x|=|q x-p|$. Thus for each $i$ less than $q$ the point $p+i x$ is closer to $(q+i) x$ than to any integer, according to the property $(c)$. Hence for all such $i$ $[p+i x]$ is equal to $[(q+i) x]$ which immediately answers the claim.

LEMMA 8. For each $u=p / q,(p, q)=1$, and $x, y$ from the interior of $J(u)$ the $c$-sequences for $x$ and $y$ coincide with the $2 q-1$ st place.

Proof. is an immediate consequence of Lemmas 6 and 7.

LeMMA 9. Let $u=p / q,(p, q)=1$, and $x$ belong to the interior of $J(u)$.

Let $i, j$ be natural numbers with $0<i<j<q$. We consider the differences $c_{j}(x)-c_{i}(x)$, $c_{j+1}(x)-c_{i+1}(x), \ldots, c_{2 q-1}(x)-c_{2 q-1+i-j}(x)$. The claim is that sequence is not constant.

Proof. We denote the difference $c_{j}(x)-c_{i}(x)$ by $d$. Then we put $z$ to be $j x-d$. Since we have $(j+m) x-d=m x+z$ it is enough to prove that the equality $c_{i+m}=d[z+m x]$ does not hold for some natural $m$ not greater than $2 q-1-j$. We assume now that $z>i x$ and $q x<p$. The three other possible cases can be dealt with in a similar way. In our case we take $m$ equal to $q-i$, in other cases you sometimes need $q-j$ instead of that. We obtain that $z+m x$ is greater than $p$, because $|z-i x| \not|p-q x|$. The last inequality is a consequence of property $(c)$ since we have:

$$
\begin{aligned}
|z-i x| & \geq \operatorname{dist}(i x, j x+\mathbb{Z})=\operatorname{dist}(\mathbb{Z}+i x, j x) \\
& =\operatorname{dist}(\mathbb{Z},(j-i) x)>\operatorname{dist}(\mathbb{Z}, q x)=|p-q x| .
\end{aligned}
$$

Hence $[z+m x]$ is not less than $p$ while $(i+m) x=q x$ has been assumed to be less than $p$.

\section{Orbits of 0 by functions $f$}

Why is the investigation of such orbits useful in our problem? Recall the assumptions of LV. They guarantee that there is a flat shelf of the graph of $f_{\lambda}$ over 0 . This is illustrated by figure 3 . It gives us the possibility to decide that $\rho(\lambda)$ is rational, $p / q$ say, knowing only the orbit of 0 by $f_{\lambda}$. Namely, if it happens that $f_{\lambda}^{q}(0)$ is between $\alpha_{\lambda}-1+p$ and $\beta_{\lambda}+p$, that is under some shelf of the graph of $f_{\lambda}$, then 0 is preperiodic and the limit of $f_{\lambda}^{n}(0) / n$ is obviously $p / q$. This gives us only a sufficient condition for the rationality of the rotation number, nevertheless estimations of the measure of $\rho^{-1}(\mathbb{Q})$ based on it are accurate enough. Observe that (2) of LV ensures that the shelves are not too short.

The main tool in studying the orbits of 0 are the sequences $\left[f_{\lambda}^{\prime}(0)\right]$. They contain information about the ordering on the circle of orbits of the image of 0 by the canonical projection by maps $F$. Since the weakly order preserving map of $S^{1}$ with irrational rotation number $x$ is topologically semi-conjugate to the rotation by $2 \pi x$, it is not difficult to see the following fact: 
If $\rho(\lambda)$ is irrational then $\left[f_{\lambda}^{i}(0)\right]=c_{i}(\rho(\lambda))$ for every natural $i$.

This fact allows us to apply the results of $\S 5$. We define sets $I(u)$ for rational numbers $u$ which are analogous to $J(u)$ from $\S 6$. Using the criterion mentioned above shall prove that each $I(u)$ contains a subinterval, called $K(u)$, consisting of parameters for which the rotation number is $u$. In the next section we shall estimate the size of $K(u)$ using the differentiability assumptions. In the present section we prove some preliminary lemmas. The most important of them is Proposition 1.

To simplify notations we introduce functions $g_{i}: T \rightarrow \mathbb{R}$ given by $g_{i}(\lambda)=f_{\lambda}^{i}(0)$ for any natural $i$.

LEMMA 10. The functions $g_{i}$ are increasing.

Proof. By the assumptions (4) and (5) of $\operatorname{LV} f_{\lambda}(x)$ increases with $\lambda$ for any $x$. Recall the function $g$ defined in (5) of LV. It is equal to $g_{1}-1$. So the claim holds for $i=1$. Now we assume that it holds for some $i$. Then we have:

$$
g_{i+1}(\lambda)=f_{\lambda}\left(g_{i}(\lambda)\right) \text { by definition. }
$$

Since $f_{\lambda}(x)$ increase with $\lambda$ and does not decrease with $x$, the function $g_{i+j}$ increases provided $g_{i}$ is such. The claim for every $i$ follows by the principle of induction.

Definition. We fix some rational $u$ expressible by the irreducible fraction of the form $p / q$. We assume that $b_{k}(u)=u$ for some odd $k$ (see $\S 5$ for the definition of $\left.b_{k}\right)$. First we define $j(u)$ by $j(u)=\rho^{-1}($ int $J(u))$. Next, let $i(u)$ be the set of parameters $\lambda$ from $T$ for which the equality $\left[f_{\lambda}^{i}(0)\right]=c_{i}(u)$ holds for any positive integer $i$ less than $2 q$ and for any $\lambda_{1}$ from $j(u) \lambda$ is greater than $\lambda_{i}$. Then the set $I(u)$ is defined to be the union of $i(u)$ and $j(u)$.

The definition of $I(u)$ is rather complicated, but the idea is simple. The $I(u)$ is nearly the same as $\rho^{-1}(J(u))$. We have proved in $\S 5$ that for numbers belonging to the same $J(u)$ their $c$-sequences have nice common properties. Now the same is true of parameters belonging to the same $I(u)$ and the sequences $\left[f_{\lambda}^{\prime}(0)\right]$.

COROLlaRy 10. For each $u=p / q$, the set $I(u)$ is an interval whose upper endpoint $s$ is characterized by $s=\inf \{\lambda \in T: \lambda \geq j(p / q)\}$ and $g_{i}(\lambda) \in \mathbb{Z}$ for some $i$ with $1 \leq i \leq$ $2 q-1$, unless $s=\sup T$.

We are not going to prove it since it is a simple consequence of Lemmas 3 and 10.

Proposition 1. Let $u=p / q$ be as required by the definition of $I(u)$. Then the interiors of $g_{i}(I(u))$ are disjoint even when projected onto $S^{1}$ by the canonical projection.

Proof. Suppose that the claim is not true, that is there exist integers $i$ and $j$, positive and not greater than $q$ and $\lambda_{1}, \lambda_{2}$ both from $I(u)$ such that $f_{\lambda_{1}}^{i}(0)=f_{\lambda_{2}}^{j}(0)-d$ for some integer $d$. Then we also can find in $I(u)$ the parameters $\lambda_{g}$ and $\lambda_{d}$ such that $\lambda_{g} \geq \lambda_{2} \geq \lambda_{d}$ and $f_{\lambda_{g}}^{i}(0) \geq f_{\lambda_{2}}^{j}(0)-d \geq f_{\lambda_{d}}^{i}(0)$. Since $f$ increases with $\lambda$ we obtain for every natural $m$ :

$$
f_{\lambda_{2}}^{i+m}(0) \geq f_{\lambda_{2}}^{m}\left(f_{\lambda_{2}}^{j}(0)-d\right)=f_{\lambda_{2}}^{j+m}(0)-d \geq f_{\lambda_{d}}^{i+m}(0) .
$$

It implies that

$$
\left[f_{\lambda_{g}}^{i+m}(0)\right] \geq\left[f_{\lambda_{2}}^{j+m}(0)\right]-d \geq\left[f_{\lambda_{d}}^{i+m}(0)\right]
$$


For $m$ not greater than $2 q-i-1$ the first and the last terms in this sequence of inequalities are equal by the definition of $I(u)$ and Lemma 8 . Thus the difference between $\left[f_{\lambda_{2}}^{j+m}(0)\right]$ and $\left[f_{\lambda_{g}}^{i+m}(0)\right]$ is $d$ for all such $m$. On the other hand, for any $\lambda$ in $I(u)$ the sequence $\left[f_{\lambda}^{i}(0)\right]$ to the $2 q-1$ st place coincides with the $c$-sequence for any $\boldsymbol{x}$ from $J(u)$. It leads us to a contradiction with the claim of Lemma 9.

Proposition 2. Let $u=p / q$ be as usual. Then for each integer $i$, positive and less than $q$, and for each $\lambda$ in $I(u)$ the fractional part of $g_{i}(\lambda)$ belongs to $\left(\beta_{\lambda}, \alpha_{\lambda}\right)$.

Proof. It is a corollary to the previous proposition. The only thing to be observed is that if for some $i$ and $\lambda$ the claim were not true then $g_{i+1}(I(u))$ would have to intersect with $g_{1}(I(u))$ on some open subset. (We mean the intersection modulo 1 or after projecting to $S^{1}$.)

Proposition 3. For $u, p, q$ as usual and $s$ being the upper endpoint of $I(u)$

$$
g_{q}(s)=p \quad \text { or } \quad s=\sup T \text {. }
$$

Proof. Recall the characterization of $s$ given in Corollary 10. Then observe that the range of $i$ in that condition can be confined to positive integers not exceeding $q$. It follows from Lemma 7. On the other hand, Proposition 2 implies that for $i$ less than $q$ the fractional part of $g_{i}(s)$ must belong to $\left(\beta_{s}, \alpha_{s}\right]$, hence $g_{i}(s)$ cannot be an integer for any such $i$. Thus if $s$ is not the supremum of the whole $T$ then the only possible $i$ is $q$. Obviously $g_{q}(s)$ cannot be greater than $p$, otherwise there would be $s^{\prime}$ less than $s$ with $g_{q}\left(s^{\prime}\right)=p$ in contradiction with the characterization of Corollary 10.

\section{Estimations}

Throughout this section $u$ will always denote a rational number from the interval $(0,1), p / q$ will be the irreducible form of $u$.

We are going to estimate the length of $i(u)$ as related to the whole $I$. Actually we shall approximate $i(u)$ by a still smaller set $k$ defined by:

$$
K=\left\{\lambda \in I:\left\{g_{q}(\lambda)\right\} \geq \alpha\right\},
$$

that is $K$ consists of these parameters whose image by $g_{q}$ is under the shelf of the graph of $f_{\lambda}$ for each $\lambda$ from $T$ (see (2) of LV). As we have already noted at the beginning of the previous section, for all parameters from $K$ the $\rho$ is certain to be u.

We already know by Proposition 3 that the ratio $\left|g_{q}(K)\right| /\left|g_{q}(I)\right|$ cannot be too small, not less than $\alpha$ anyway. To get the similar estimation of the ratio $K(u) / I(u)$ we use the assumptions involving differentiability conditions for $f$. The result of these computations is given by Lemma 11 .

Before we formulate it we introduce some notations. The numbers $u, p, q$ are fixed so we shall write $K$ for $K(u)$. The supremum of $K$ will be denoted by $s$, the infimum by $r$. Then, we choose some $t$ in $I(u) \backslash \bar{K}$. The interval $(t, r)$ will be denoted by $W$. LEMMA 11. There exists a finite constant $C$, independent of $u$ and $t$ chosen as described above such that:

$$
\frac{\left|g_{q}(K)\right|}{\left|g_{q}(W)\right|} \leq C \frac{|K|}{|W|}
$$


Proof. The numbers $z_{i}$ for $i=1, \ldots, q-1$ are defined by

$$
z_{i}=\max \left(1, \sup \frac{f_{t}^{\prime}\left(x_{1}\right)}{f_{t}^{\prime}\left(x_{2}\right)}\right)
$$

where the supremum is taken over $x_{1}$ and $x_{2}$ both belonging to $g_{i}(K \cup W)$.

We are going to prove by induction with respect to $i=1, \ldots q$ the following fact:

$$
\frac{\left|g_{i}(K)\right|}{\left|g_{i}(W)\right|} \leq \frac{|K|}{|W|} B \frac{L}{\sigma}(1+2 L(r-t))^{i-1} \prod_{1 \leq j<i} z_{j} .
$$

The right-hand side of the above inequality will be denoted by $c_{i}$. The numbers $L$ and $\sigma$ are defined by (3) and (4) of LV while $B$ is

$$
\frac{\sup \left\{\frac{d g}{d \lambda}(\lambda): \lambda \in T\right\}}{\inf \left\{\frac{d g}{d \lambda}(\lambda): \lambda \in T\right\}}
$$

it is finite by (5) of LV.

For $i=1$ we obtain easily that $\left|g_{i}(K)\right| /\left|g_{i}(W)\right| \leq B|K| /|W|$. Since $L / \sigma$ is greater than 1 the inequality holds. Let it hold for some $i<q$. We denote $f_{t}\left(g_{i}(t)\right), f_{t}\left(g_{i}(r)\right)$ and $f_{i}\left(g_{i}(s)\right)$ by $\bar{t}, \bar{r}, \bar{s}$ respectively. By the mean value theorem it is easy to see that

$$
\frac{\bar{s}-\bar{r}}{\bar{r}-\bar{t}} \leq \frac{\left|g_{i}(K)\right|}{\left|g_{i}(W)\right|} z_{i}
$$

Let the numbers $D_{1}, D_{2}, D_{3}$ be defined by:

$$
\begin{aligned}
& D_{1}=g_{i+1}(r)-\bar{r}, \\
& D_{2}=g_{i+1}(s)-f_{r}\left(g_{i}(s)\right), \\
& D_{3}=g_{i+1}(s)-\bar{s}-D_{2}-D_{1} .
\end{aligned}
$$

Observe that $D_{1}$ and $D_{2}$ and necessarily positive while $D_{3}$ may be negative. We have:

$$
g_{i+1}(r)=\bar{r}+D_{1}, \quad g_{i+1}(s)=D_{1}+D_{2}+D_{3}+\bar{s} \text { and } g_{i+1}(t)=\bar{t} .
$$

We consider the interval $\left(g_{i+1}(r), g_{i+1}(s)-D_{3}\right)$ denoted by $\tilde{K}$. Then we obtain the following relations:

$$
\left|g_{i+1}(W)\right|=|\bar{r}-\bar{t}|+D_{1}, \quad|\tilde{K}|=|\bar{s}-\bar{r}|+D_{2}, \quad\left|g_{i+1}(K)\right|=|\tilde{K}|+D_{3}
$$

First we compute

$$
\frac{|\tilde{K}|}{\left|g_{i+1}(W)\right|} \leq \max \left(\frac{\left|g_{i}(K)\right|}{\left|g_{i}(W)\right|} z_{i}, \frac{D_{2}}{D_{1}}\right)
$$

by $*$. By the mean value theorem $D_{2} / D_{2}$ cannot exceed

$$
\frac{(s-r) \sup \partial f / \partial \lambda}{(r-t) \inf \partial f / \partial \lambda}=\frac{|K|}{|W|} \frac{L}{\sigma} .
$$

On the other hand, by the inductional hypothesis,

$$
\frac{\left|g_{i}(K)\right|}{\left|g_{i}(W)\right|} \leq c_{i} \frac{|K|}{|W|} .
$$


We obtain thus:

$$
\frac{|\tilde{K}|}{\left|g_{i+1}(W)\right|} \leq \frac{|K|}{|W|} \max \left(c_{i} z_{i}, \frac{L}{\sigma}\right)=\frac{|K|}{|W|} c_{i} z_{i}
$$

since $c_{i}>L / \sigma$ and $z_{i} \geq 1$. We have

$$
\frac{\left|g_{i+1}(K)\right|}{\left|g_{i+1}(W)\right|}=\frac{\left|g_{i+1}(K)\right|}{|\tilde{K}|} \frac{|\tilde{K}|}{\left|g_{i+1}(W)\right|} \leq \frac{\left|g_{i+1}(K)\right|}{\left|g_{i+1}(K)\right|-D_{3}} c_{i} z_{i} \frac{|K|}{|W|}
$$

To prove the inequality for $i+1$ we need only the estimation

$$
\frac{\left|g_{i+1}(K)\right|}{\left|g_{i+1}(K)\right|-D_{3}} \leq 1+2 L(r-t) \text {. }
$$

We assume that $D_{3}$ is positive and calculate it as follows:

$$
\begin{aligned}
D_{3} & =g_{i+1}(s)-\bar{s}-D_{1}-D_{2}=g_{i+1}(s)-\bar{s}-\left(g_{i+1}(s)-f_{r}\left(g_{i}(s)\right)\right)-D_{1} \\
& =f_{r}\left(g_{i}(s)\right)-\bar{s}-\left(f_{r}\left(g_{i}(r)\right)-\bar{r}\right) \\
& =\int_{t}^{r} \frac{\partial f}{\partial \lambda}\left(y, f_{y}\left(g_{i}(s)\right)\right) d y-\int_{t}^{r} \frac{\partial f}{\partial \lambda}\left(y, f_{y}\left(g_{i}(r)\right)\right) d y .
\end{aligned}
$$

We denote by $r_{0}$ the least number from the interval $(t, r)$ such that

$$
\int_{1}^{r_{0}}\left(\frac{\partial f}{\partial \lambda}\left(y, f_{y}\left(g_{i}(s)\right)\right)-\frac{\partial f}{\partial \lambda}\left(y, f_{y}\left(g_{i}(r)\right)\right)\right) d y=D_{3} .
$$

Then we have;

$$
D_{3} \leq \int_{t}^{r_{0}} L\left|f_{y}\left(g_{i}(s)\right)-f_{y}\left(g_{i}(r)\right)\right| d y
$$

where $L$ stands for the upper bound of $\partial^{2} f / \partial x \partial \lambda$ over $T \times \mathbb{R}$. By the definition of $r_{0}$ we obtain for $y$ between $t$ and $r_{0}$ that

$$
D_{3}>f_{y}\left(g_{i}(s)\right)-\vec{s}-f_{y}\left(g_{i}(r)\right)+\vec{r}
$$

thus

$$
\left|f_{y}\left(g_{i}(s)\right)-f_{y}\left(g_{i}(r)\right)\right| \leq D_{3}+\bar{s}-\bar{r}=\left|g_{i+1}(K)\right|-D_{2}<\left|g_{i+1}(K)\right| .
$$

When we use this inequality we get

$$
D_{3} \leq\left(r_{0}-t\right) L\left|g_{i+1}(K)\right| \leq L(r-t)\left|g_{i+1}(K)\right| .
$$

Further we also obtain:

$$
\frac{\left|g_{i+1}(K)\right|}{\left|g_{i+1}(K)\right|-D_{3}} \leq \frac{1}{1-L(r-t)} .
$$

By (3) of LV $L(r-t)<\frac{1}{2}$ and we obtain

$$
\frac{1}{1-L \cdot(r-t)} \leq 1+2 L(r-t)
$$

which is exactly what we need to complete the proof of the inequality for $i+1$.

In particular we get

$$
\frac{\left|g_{0}(K)\right|}{\left|g_{q}(W)\right|} \leq B \frac{L}{\sigma}\left(\prod_{1 \leq j<q} z_{j}\right)(1+2 L(r-t))^{q-1} \frac{|K|}{|W|}
$$


We must prove that $c_{q}$ can be estimated independently of $u$ and $t$. First we are going to estimate $z_{i}$. Observe that, by Proposition 2, $g_{i}(K \cup W)$ is always contained modulo 1 in the interval $\left(\beta_{t}, 1\right)$. We distinguish 2 cases:

(1) $\left\{g_{i}(t)\right\}>\gamma_{r}$. Then, by (6)(a) of LV, $z_{i}$ is 1 .

(2) Otherwise, by (6)(b) of LV, the denominator of the fraction $f_{t}\left(x_{1}\right) / f_{t}\left(x_{2}\right)$ is separated from 0 by the constant $\zeta$ if only $x_{2}$ is less than $\gamma_{t}$ modulo 1 . If it is not the case, then this ratio does not exceed 1 . Hence

$$
\frac{f_{t}^{\prime}\left(x_{1}\right)}{f_{t}^{\prime}\left(x_{2}\right)} \leq 1+\frac{1}{\zeta} f_{t}^{\prime \prime}(\tilde{x})\left|x_{1}-x_{2}\right|
$$

for some $\tilde{x}$ between $x_{1}$ and $x_{2}$. Further, since $f_{t}^{\prime \prime} \leq L$ we obtain

$$
z_{i} \leq 1+\frac{L}{\zeta}(s-t)
$$

This estimation works in both cases.

Now we are able to estimate the product of $z_{j}$. Since

$$
z_{j} \leq \exp \left(\frac{L}{\zeta}(s-t)\right)
$$

for each $j$ less than $q$, their product does not exceed

$$
\exp \left(\frac{L}{\zeta} q|I(u)|\right)
$$

In the analogous way we can estimate $(1+2 L(r-t))^{q-1}$ by $\exp (2 L q|I(u)|)$. Finally we get that $c_{q}$ is not greater than

$$
B \frac{L}{\sigma} \exp \left(2 L+\frac{L}{\zeta}\right) \exp (q|I(u)|) .
$$

It remains to show that $q|I(u)|$ is bounded by some constant independent of $u$. It is provided by the following lemma:

LEMMA 12. For each $u, q|I(u)| \leq \sigma^{-1}$.

Proof. Proof of Lemma 12:

First of all we notice that $\mathrm{d} g / \mathrm{d} \lambda i \geq \sigma$ for every natural $i$ and parameter value $\lambda$. It is true for $i=1$ by (5) of LV. Then we proceed by induction:

$$
\frac{\mathrm{d} g_{i+1}}{\mathrm{~d} \lambda}=\frac{\partial f_{\lambda}}{\partial x} \frac{\mathrm{d} g_{i}}{\mathrm{~d} \lambda}+\frac{\partial f}{\partial \lambda}
$$

- the first term is non-negative and the second is not less than $\sigma$. It implies that for each $u$ the length of $g_{i}(I(u))$ is at least $\sigma|I(u)|$. The total of these lengths for $i$ between 1 and $q$ is thus not less than $q \sigma|I(u)|$. On the other hand, it cannot exceed 1 by Proposition 1. The claim of the lemma follows.

The proof of Lemma 11 is also completed.

Proposition 4. For each $u$ if only $\sup (I(u))<\sup T$, then the ratio $|K(u)| /|I(u)|$ is at least $(1-\alpha) /(C+1)=w$.

Proof. We begin with the remark that, since the constant $C$ of Lemma 11 does not 
depend on $t$, the ratio $|K(u)| /|I(u) \backslash K(u)|$ is bounded by the analogous ratio of lengths of the images by $g_{q}$ multiplied by the same constant $C$. By Proposition 3 the length of $g_{q}(K(u))$ is at least $1-\alpha$ and the length of the whole $g_{q}(I(u))$ must be less than 1 . Hence $|K(u)| /(|I(u)|-|K(u)|)$ is not less than $(1-\alpha) / C$. After the simple calculation the claim of the proposition follows.

\section{The completion of the proof of $L V$}

Now we are ready to finish the proof of LV. Suppose by the contradiction that the set $S=\rho^{-1}(\mathbb{R} \backslash \mathbb{Q})$ has positive measure and let $y$ be its point of density. We may assume that $y<\sup \rho(T)$ since by Lemma $12 \rho^{-1}(\sup \rho(T))$ is at most one point when $\sup \rho(T)$ is irrational. Next, we consider the sequence of intervals $I_{n}$ defined by $I_{n}=I\left(b_{2 n+1}(\rho(y))\right)$. They all contain $y$ and their lengths tend to zero by Lemma 12. Moreover, for $n$ sufficiently large sup $I_{n}<\sup T$ and the use of the Proposition 4 results in the contradiction with the definition of $y$. This completes the proof of LV.

\section{REFERENCES}

[1] L. Alsedá, J. Llibre, M. Misiurewicz \& C. Simó. Twist periodic orbits and topological entropy for continuous maps of the circle of degree one which have a fixed point. Ergod. Th. \& Dynam. Sys. 5 (1985), 501-517.

[2] V. I. Arnold. Small denominators, I. Mappings of the circumference onto itself. AMS Translations 2 (46) (1965), 213-284.

[3] G. H. Hardy \& E. M. Wright. An Introduction to the Theory of Numbers Chapter X Oxford: Clarendom Press, 1945.

[4] M. Herman. Mesure de Lebesgue et nombre de rotation, geometry and Topology. Springer Lecture Notes in Mathematics 597 (1977), 271-293.

[5] O. E. Lanford III. A numerical study of the likelihood of phase locking. Physica 14D (1985), 403-408.

[6] M. Misiurewicz. Rotation Interval for a Class of Maps of the Real Line into Itself. Institut mittag: Leffler, Report No. 10, 1984.

[7] S. Newhouse, J. Palis \& F. Takens. Bifurcations and stability of families of diffeomorphisms Publ. Mathem. IHES 57 (1983). 